\title{
Primary central nervous system ALK-negative anaplastic large cell lymphoma: a case report and literature review
}

\author{
Changwei Yuan ${ }^{1} \wedge$, Hongzhou Duan ${ }^{1 \wedge}$, Yingjin Wang ${ }^{1}$, Jiayong Zhang $^{1}$, Jinping Ou $^{2}$, Wei Wang ${ }^{3}$, \\ Min Zhang ${ }^{4}$
}

${ }^{1}$ Department of Neurosurgery, Peking University First Hospital, Beijing, China; ${ }^{2}$ Department of Hematology, Peking University First Hospital, Beijing, China; ${ }^{3}$ Department of Pathology, Peking University First Hospital, Beijing, China; ${ }^{4}$ Department of Radiotherapy, Peking University First Hospital, Beijing, China

Correspondence to: Hongzhou Duan. Department of Neurosurgery, Peking University First Hospital, No. 8 Xishiku Street, Xicheng District, 100034, Beijing, China. Email: duanhongzhou@126.com.

\begin{abstract}
Primary central nervous system anaplastic lymphoma kinase (ALK)-negative anaplastic large cell lymphoma (ALCL) is an extremely rare type of primary central nervous system lymphoma (PCNSL). There are only nine cases reported in the literature to date, most of which have an overall survival time of no more than 8 months. Herein, we report such a rare case who has a good outcome with the longest survival time and perform a review of the literature. A 19-year-old male patient was admitted to the hospital complaining of dizziness. CT and MRI imaging showed a heterogeneous enhanced lesion in the left parieto-occipital lobe and the leptomeninges of the occipital lobe and the cerebellum. The lesion was resected and confirmed to be ALK-negative ALCL by pathological examination. Then, the patient received 10 cycles of chemotherapy with high-dose methotrexate (HD-MTX) and whole-brain radiotherapy. The patient recovered well and was regularly followed up. He was free of symptoms without recurrence on imaging examination 3 years later. ALCL is a rare type of PCNSL. HD-MTX combined with radiation is an effective therapeutic approach. However, further prospective studies with a large number of patients are needed to identify the biological characteristics of this rare type of PCNSL.
\end{abstract}

Keywords: Anaplastic large cell lymphoma (ALCL); anaplastic lymphoma kinase (ALK); primary central nervous system lymphomas (PCNSLs); treatment; case report

Submitted Mar 09, 2021. Accepted for publication May 21, 2021.

doi: 10.21037/apm-21-557

View this article at: https://dx.doi.org/10.21037/apm-21-557

\section{Introduction}

Primary central nervous system lymphomas (PCNSLs) are non-Hodgkin's lymphomas that account for approximately $3-6 \%$ of all primary intracranial tumors. The majority of PCNSLs are diffuse large B-cell lymphomas, while primary T-cell lymphoma accounts for only $2-8.5 \%$ (1). Anaplastic large cell lymphoma (ALCL) is a rare type of T-cell lymphoma. The lymphoma cells usually express T-cell markers, the activation antigen CD30 and a translocation involving the anaplastic lymphoma kinase (ALK) gene.
According to the WHO Classification, ALCL is divided into ALK-positive and ALK-negative ALCL. Primary ALKnegative patients are rarer than ALK-positive patients in the literature, and most ALK-negative PCNSL patients display variable imaging characteristics, aggressive clinical features and unfavorable outcomes with an overall survival time of no more than 8 months (2). However, herein, we present a case of an ALK-negative ALCL patient who underwent successful treatment of extensive resection followed by high-dose methotrexate (HD-MTX) and whole brain

^ ORCID: Changwei Yuan, 0000-0001-6256-8775; Hongzhou Duan, 0000-0002-6456-2307. 

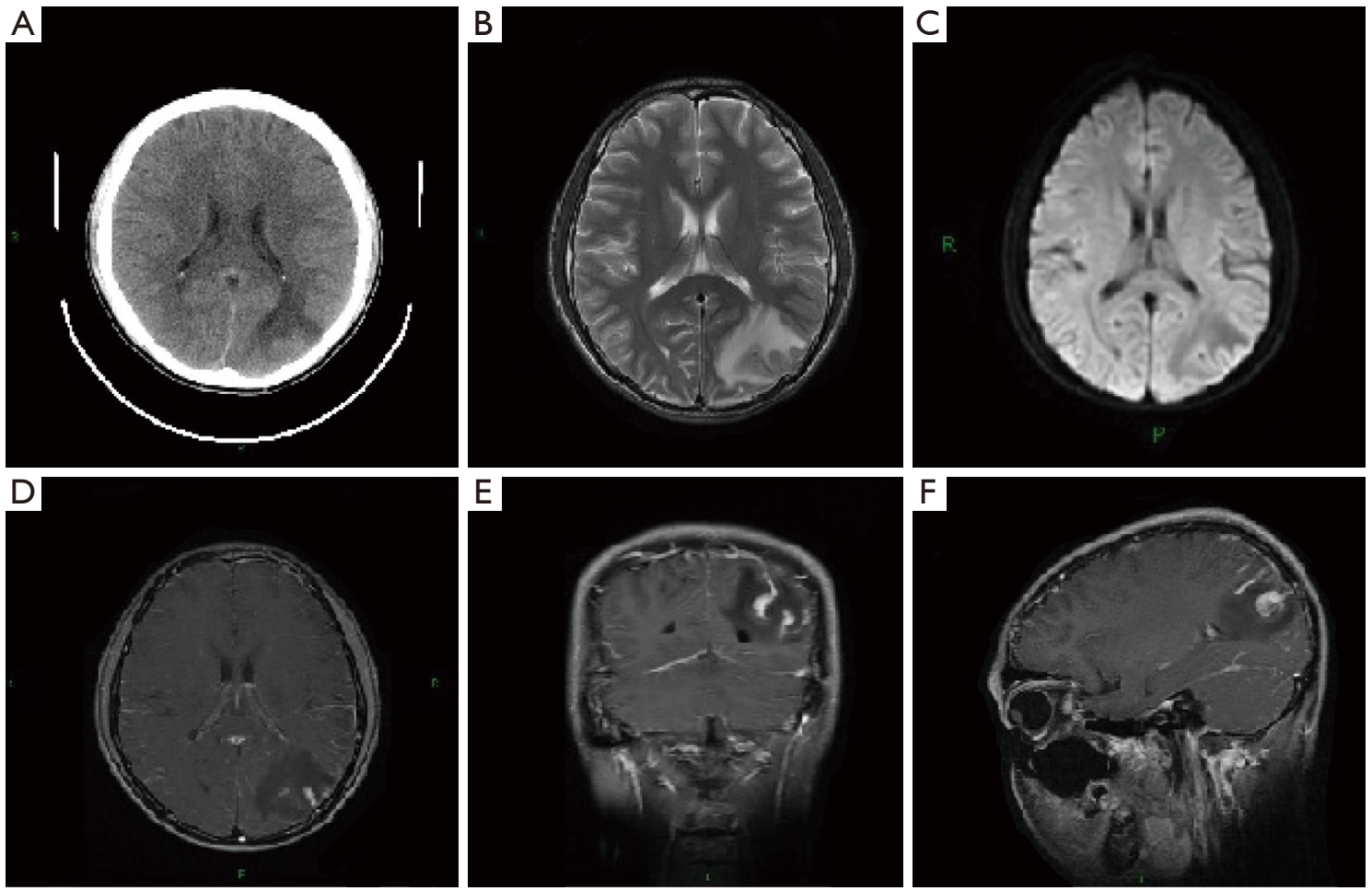

Figure 1 The preoperative images of the patient. The CT scan (A) shows a low-density lesion in the left parieto-occipital lobe. MRI shows that the lesion is hyperintense on T2-weighted images (B) and hypointense in diffuse-weighted images (C), which is partially enhanced (D, axial image; E, coronal image; F, sagittal image). The enhanced regions are located in the sulcus of the parieto-occipital lobe and the leptomeninges of the occipital lobe and the cerebellum.

radiotherapy (WBR) and achieved no evidence of disease recurrence over 36 months. We present the following case in accordance with the CARE reporting checklist (available at https://apm.amegroups.com/article/view/10.21037/apm$21-557 / \mathrm{rc})$.

\section{Case presentation}

A 19-year-old male patient was admitted to the hospital with a 10-day history of dizziness and blurred vision. Past medical history was not suggestive of intracranial pathology, and there was no presence of fever, night sweats, and weight loss, recorded at hospital admission. On physical examination, the patient appeared well, and there were no palpable cervical, axillary, or inguinal lymph nodes. Laboratory examination revealed a normal complete blood cell count with no leukocytosis. Lumbar puncture showed a slightly elevated intracranial pressure $\left(19 \mathrm{~cm} \mathrm{H}_{2} \mathrm{O}\right)$, and cerebrospinal fluid (CSF) examination showed strongly increased protein $(418 \mathrm{mg} / \mathrm{dL}$, normal $15-45 \mathrm{mg} / \mathrm{dL})$ and decreased glucose $(0.12 \mathrm{mmol} / \mathrm{L}$, normal $2.5-4.5 \mathrm{mmol} / \mathrm{L})$. No abnormal cells were present on CSF cytology, and extensive microbiological examinations of the CSF, including testing for mycobacterium tuberculosis and fungus, were all normal. Brain CT demonstrated lowdensity lesions in the left parietooccipital lobe and MRI imaging showed a heterogeneous enhanced lesion in the left parieto-occipital lobe and the leptomeninges of the occipital lobe and the cerebellum (Figure 1). Atypical infection or tumor was suspected, and lesion resection was indicated. During the operation, the lesion was located in the subcortical region and circled the vessels in the sulcus. It was crispy, easy bleeding and was indicated to be a malignant tumor by intraoperative frozen pathology. Extensive tumor resection was achieved.

Histological tissue analysis revealed that the tumor had an abnormal lymphoid-cell morphology, consisting of vesicular nuclei and prominent nucleoli as well as moderate amphophilic to eosinophilic cytoplasm (Figure 2A). In situ hybridization (ISH) of EB virus DNA is negative. 

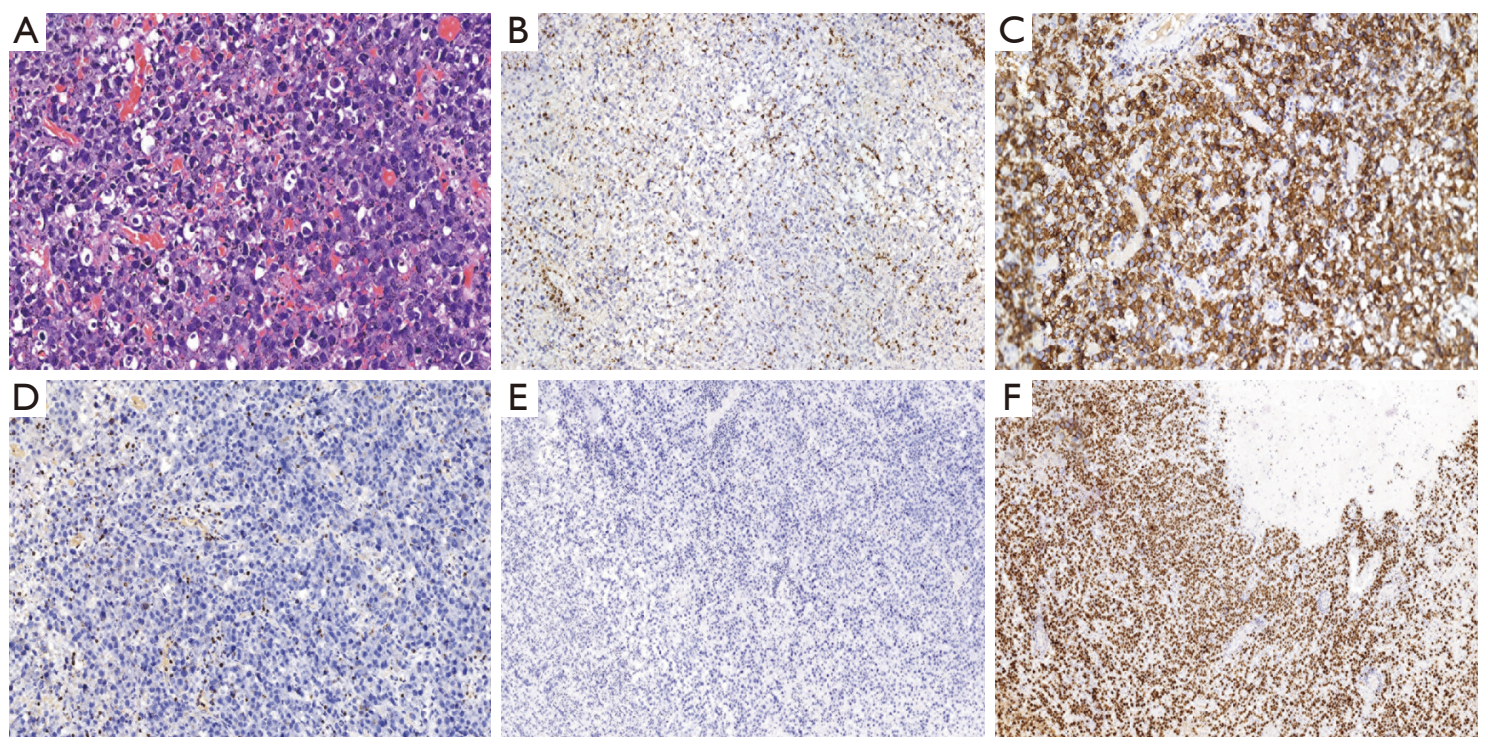

Figure 2 The pathological features of the tumor. The tumor cells have an abnormal lymphoid-cell morphology, consisting of vesicular nuclei and prominent nucleoli (A, H\&E $\times 400)$. Immunohistological staining shows that the tumor is negative for CD3 (B, $\times 200)$ and TIA1 (D, $\times 200)$ but positive for CD30 (C, $\times 200)$. ALK staining was negative $(\mathrm{E}, \times 100)$, and the Ki67 labeling index was nearly $100 \%(\mathrm{~F}, \times 100)$.

Immunohistochemical staining was performed on paraffinembedded tissue. Monoclonal antibodies specific to the following antigens were used: $\mathrm{CK}$ (AE1/AE3), vimentin, GFAP, LCA, CD43, CD3, CD20, PAK5, CD56, CD30, ALK (IA4), CD2, CD7, CD4, CD8, CD68 (KP1), granzyme B, TIA1, T-bet, and EBER-ISH. Tumor cells were positive for vimentin, LCA, CD30, CD43, CD56, CD68 (KP1), granzyme B and TIA1. The Ki67 proliferation index was almost $100 \%$. TCRB and TCRG antigen receptor gene rearrangement cloning test results were positive. Prognostically important ALK was negative (Figure 2B-2F).

The pathological diagnosis of ALK-negative ALCL was finally confirmed. Then, he was referred to a hematologist and radiation therapist. Extensive examinations did not reveal any signs of dissemination. Bone marrow biopsy was negative. Chemotherapy was applied during a 12-month period in ten cycles with the following protocol: HD-MTX $(5 \mathrm{~g} / \mathrm{msq}$, for a total of $7.8 \mathrm{~g}$ ) delivered intravenously on day 1 , methotrexate serum levels were monitored 12, 24 and 48 hours after its administration, and leucovorin calcium was used when necessary. Meanwhile, in the first, sixth, seventh, eighth, and ninth cycles of chemotherapy, intrathecal injections of $5 \mathrm{mg}$ dexamethasone and $15 \mathrm{mg}$ methotrexate were performed. Between the fourth and fifth cycles, radiotherapy was carried out with fractionated low-dose whole-brain irradiation (23.4 Gy separated in 13 fractions of 1.8 Gy each). The patient was regularly followed-up, and at the 1-year and 3-year follow-up, he was doing well with no radiological or clinical signs of tumor recurrence (Karnofsky performance scale: 90) (Figures 3,4).

All procedures performed in this study were in accordance with the ethical standards of the institutional and/or national research committee(s) and with the Helsinki Declaration (as revised in 2013). Written informed consent was obtained from the patient for publication of this case report and accompanying images. A copy of the written consent is available for review by the editorial office of this journal.

\section{Discussion}

ALCL is an exceptionally rare type of T-cell lymphoma among PCNSLs. To the best of our knowledge, only 9 cases of ALK-negative ALCL in the CNS have been clearly reported in the literature (3-10). However, the clinical and imaging manifestations of these rare cases are different, which makes the diagnosis of the disease more difficult, just like our case.

In the literature, ALCL patients can be classified into three types according to the characteristics on MRI. In the vast majority of cases, ALCL has a characteristic dense and 

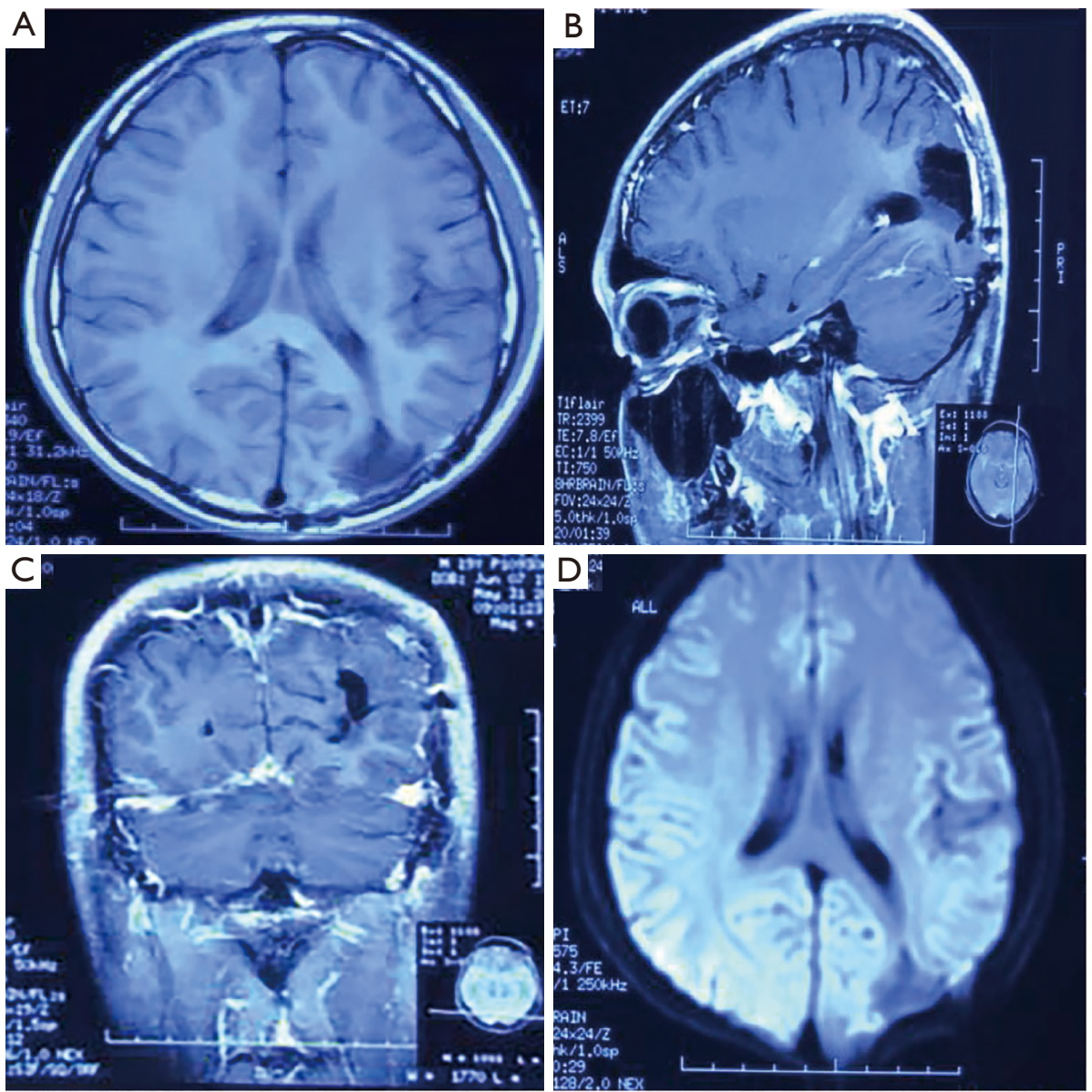

Figure $3 \mathrm{MRI}$ images at the 1-yr follow-up. There is no recurrence of tumor. (A, T1-weighted image; B, sagittal enhanced T1-weighted image; C, coronal enhanced T1-weighted image; D, DWI image). DWI, diffusion-weighted imaging.

homogeneous enhancement pattern on MRI, similar to meningioma, metastasis, glioma, etc (11). The second type of ALCL can be determined from an extreme enhancement of the lesion around the sulcus and an enhancement of the leptomeninges, which present similarly to meningitis or inflammation (12). The third type exhibits a rare condition that was characterized by a slight enhancing on MRI indicating the diffuse white matter being infiltrated (13). Our case was consistent with the second type, exhibiting a diffusely enhanced signal in the cortex of the parietooccipital lobe and an enhancement of the leptomeninges, which indicates the tumor cells disseminated by the CSF.

The treatment of ALCL cases is controversial. Although the NCCN guidelines do not recommend extended resection for PCNSL patients except chemotherapy and radiotherapy after biopsy, we also believe that it is reasonable to perform extensive resection for those patients who cannot be identified as PCNSL before and during operation, especially in such ALCL cases, because the reaction of chemotherapy and radiotherapy in ALCL cases is heterogeneous (2). Although the standard chemotherapy regimen CHOP is widely used in extranodal non-Hodgkin lymphoma, it has not been proven to be effective against PCNSL. HD-MTX seems to be a successful medication, as it is reported with better survival: of the 11 reported ALCL patients who received MTX-based chemotherapy, 9 exhibited no evidence of tumor recurrence during the follow-up (2). However, good reaction to MTX and good clinical outcomes seem to favor the ALK-positive ALCL patients.

ALK-negative ALCL patients demonstrate different clinical features and prognoses with the ALK-positive ALCL patients. In the literature, ALK-positive tumors occurred in patients much younger and had a better reaction to chemotherapy resulting in better outcome (2). 

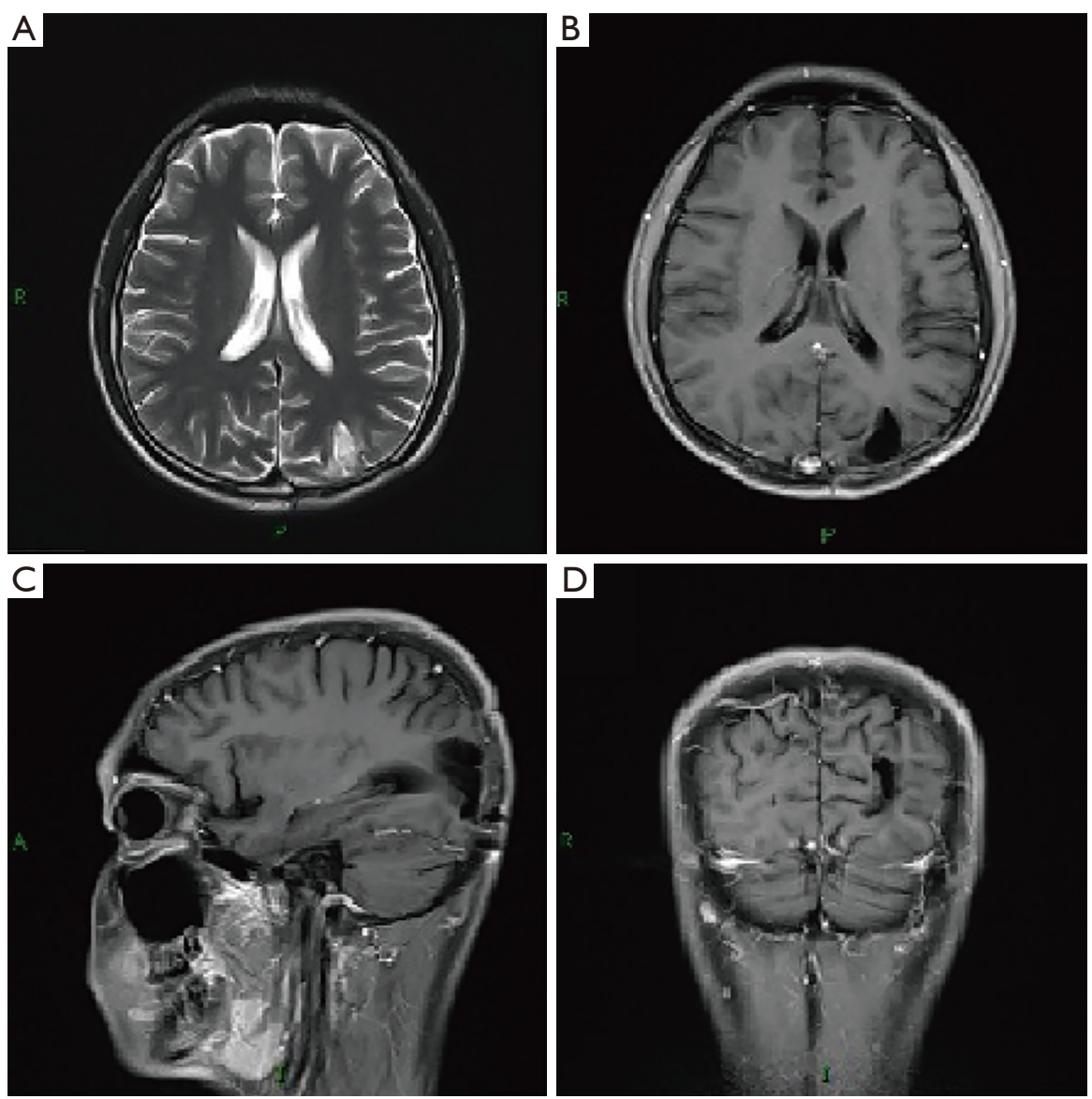

Figure 4 MRI images at the 3-yr follow-up. The lesion is resected, leaving a cavity with fluid signal in T2-weighted image (A). There is no abnormally enhanced part in the enhanced images (B, axial image; C, sagittal image; D, coronal image).

Age, LDH serum level, performance status, CSF protein concentration, and the involvement of deep structures of the brain are thought to be independent predictors of survival. We summarized all published cases of ALKnegative ALCL in the literature in Table 1, including our case. Among all patients, our case was the youngest (range 19-82 years, median 64). Most of the tumors were located in the supratentorial (80\%) area. A single lesion or multiple lesions were observed on preoperative MRI examination in 5 patients respectively. Most ALK-negative patients presented with a poor prognosis. Seven of the ten ALK-negative patients succumbed after excision with survival times ranging from 4 days to 8 months. Patients who did not receive MTX therapy (7 in 8 ) had a poor prognosis with rapid tumor recurrence. One case treated with an extensive resection followed by radiotherapy (4), and another case received a combined treatment of chemotherapy with HD-MTX and WBR (6), they all had a good outcome. In our case, the patient received maximal resection of the lesion, multiple cycles of chemotherapy and WBR, and finally achieved a best outcome without recurrence for over 36 months, compared with previous literatures. Furthermore, as there was wide leptomeninges enhancement indicating CSF dissemination, the patient received four cycles of an intrathecal injection of MTX. This might be another reason that the patient survived without tumor recurrence. However, we also admit that high-dose MTX and WBR may cause greater neurotoxicity and long-term injury, so such patients must receive long-term follow-up and timely treatment. With the increase of such cases and rich treatment experience, the appropriate dose of MTX and radiation dose and range will be further determined.

To the best of our knowledge, we report the youngest case of ALK-negative ALCL of the CNS with the best outcome. Although most cases of ALK-negative ALCL in 
Table 1 Reported cases with ALK-negative anaplastic large-cell lymphoma of PCNL in literature

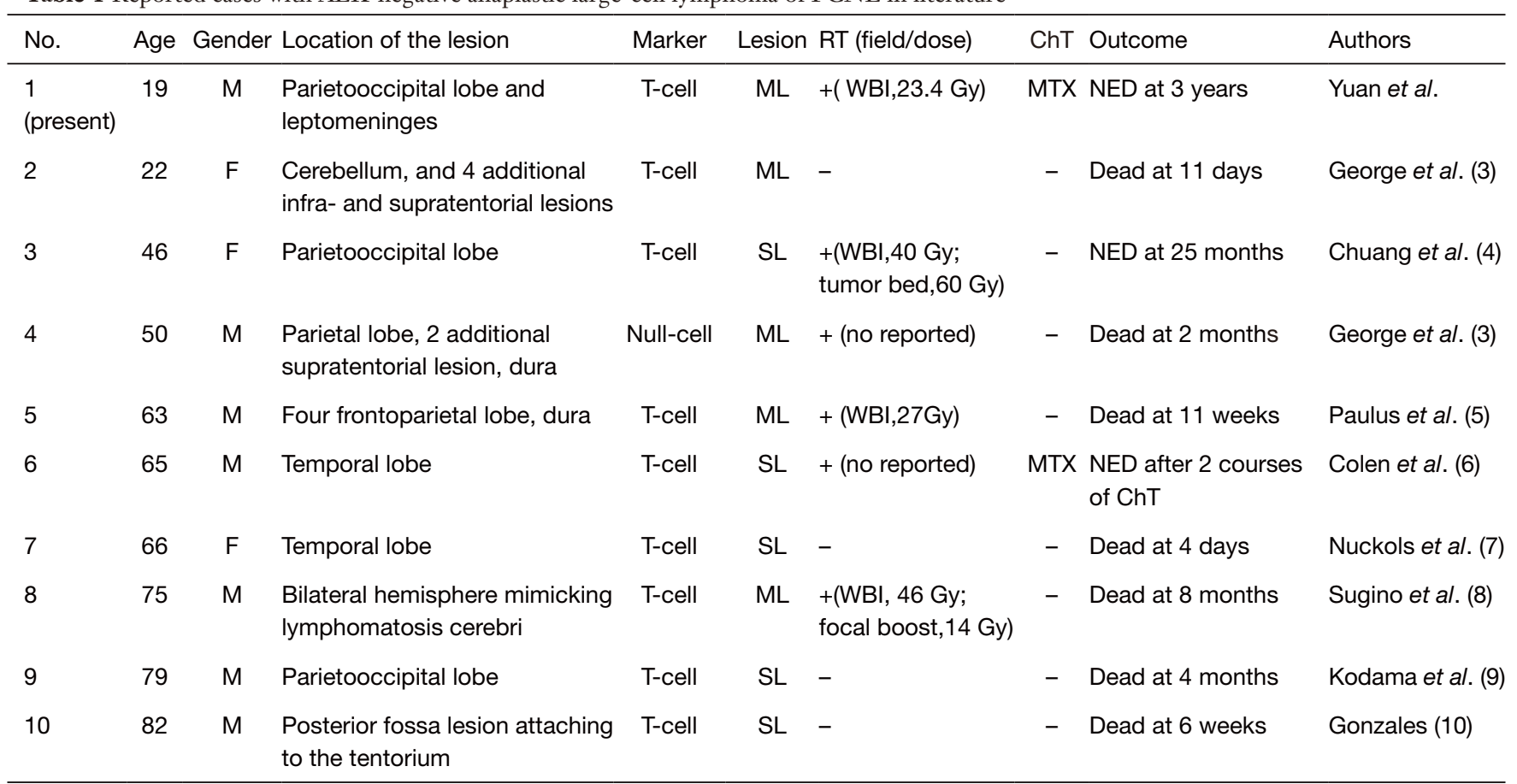

ALK, anaplastic lymphoma kinase; PCNL, primary central nervous lymphomas; RT, radiotherapy; ChT, chemotherapy; F, female; M, male; ML, multiple lesions; MTX, methotrexate; NED, no evidence of disease; SL, single lesion; WBI, the whole brain irradiation.

the literature have a poor prognosis, we advocate extensive resection, aggressive chemotherapy, including intravenous and intrathecal administration of high-dose MTX followed by radiotherapy, which might lead to a good outcome without tumor recurrence. However, future studies including large numbers of cases are required.

\section{Acknowledgments}

Funding: This work was supported by the Chinese National Nature Science Foundation (Grant No. 81541119), Youth Clinical Research Project of Peking University First Hospital (Grant No.2019CR02) and Peking University First Hospital Cross Clinical Study Foundation.

\section{Footnote}

Reporting Checklist: The authors have completed the CARE reporting checklist. Available at https://apm.amegroups. com/article/view/10.21037/apm-21-557/rc

Conflicts of Interest: All authors have completed the
ICMJE uniform disclosure form (available at https://apm. amegroups.com/article/view/10.21037/apm-21-557/coif). The authors have no conflicts of interest to declare.

Ethical Statement: The authors are accountable for all aspects of the work in ensuring that questions related to the accuracy or integrity of any part of the work are appropriately investigated and resolved. All procedures performed in this study were in accordance with the ethical standards of the institutional and/or national research committee(s) and with the Helsinki Declaration (as revised in 2013). Written informed consent was obtained from the patient for publication of this case report and accompanying images. A copy of the written consent is available for review by the editorial office of this journal.

Open Access Statement: This is an Open Access article distributed in accordance with the Creative Commons Attribution-NonCommercial-NoDerivs 4.0 International License (CC BY-NC-ND 4.0), which permits the noncommercial replication and distribution of the article with the strict proviso that no changes or edits are made and the 
original work is properly cited (including links to both the formal publication through the relevant DOI and the license). See: https://creativecommons.org/licenses/by-nc-nd/4.0/.

\section{References}

1. Ferreri AJ, Reni M, Pasini F, et al. A multicenter study of treatment of primary CNS lymphoma. Neurology 2002;58:1513-20.

2. Nomura M, Narita Y, Miyakita Y, et al. Clinical presentation of anaplastic large-cell lymphoma in the central nervous system. Mol Clin Oncol 2013;1:655-60.

3. George DH, Scheithauer BW, Aker FV, et al. Primary anaplastic large cell lymphoma of the central nervous system: prognostic effect of ALK-1 expression. Am J Surg Pathol 2003;27:487-93.

4. Chuang SS, Huang W, Lin CN, et al. Primary cerebral anaplastic large cell lymphoma containing abundant reactive histiocytes and eosinophils. A case report and literature review. Pathol Res Pract 2001;197:647-52.

5. Paulus W, Ott MM, Strik H, et al. Large cell anaplastic (KI-1) brain lymphoma of T-cell genotype. Hum Pathol 1994;25:1253-6.

Cite this article as: Yuan C, Duan H, Wang Y, Zhang J, Ou J, Wang $W$, Zhang M. Primary central nervous system ALKnegative anaplastic large cell lymphoma: a case report and literature review. Ann Palliat Med 2022;11(4):1554-1560. doi: 10.21037/apm-21-557
6. Colen CB, Rayes M, Kupsky WJ, et al. Synchronous meningioma and anaplastic large cell lymphoma. Neuropathology 2010;30:260-6.

7. Nuckols JD, Liu K, Burchette JL, et al. Primary central nervous system lymphomas: a 30-year experience at a single institution. Mod Pathol 1999;12:1167-73.

8. Sugino T, Mikami T, Akiyama Y, et al. Primary central nervous system anaplastic large-cell lymphoma mimicking lymphomatosis cerebri. Brain Tumor Pathol 2013;30:61-5.

9. Kodama K, Hokama M, Kawaguchi K, et al. Primary ALK-1-negative anaplastic large cell lymphoma of the brain: case report and review of the literature. Neuropathology 2009;29:166-71.

10. Gonzales M. Primary meningeal anaplastic large cell lymphoma. Pathology 2003;35:451-2.

11. Muly S, Liu S, Lee R, et al. MRI of intracranial intraventricular lesions. Clin Imaging 2018;52:226-39.

12. Lamszus K. Meningioma pathology, genetics, and biology. J Neuropathol Exp Neurol 2004;63:275-86.

13. Carlson BA. Rapidly progressive dementia caused by nonenhancing primary lymphoma of the central nervous system. AJNR Am J Neuroradiol 1996;17:1695-7. 